\title{
28 Research Square \\ LncRNAs Function as Critical Metabolic Regulators in yak Liver by Integrative Transcriptome Analysis
}

Wei Xia

Southwest Minzu University

Fang Fu

Southwest Minzu University

Li Wang ( $\nabla$ qinxin916@aliyun.com)

Southwest Minzu University https://orcid.org/0000-0001-8267-9309

Xiaolin Luo

Southwest Minzu University

Jiuqiang Guan

Southwest Minzu University

Research article

Keywords: LncRNAs, Yak, Liver, Integrative transcriptome

Posted Date: July 28th, 2020

DOI: https://doi.org/10.21203/rs.3.rs-32613/v1

License: (9) This work is licensed under a Creative Commons Attribution 4.0 International License.

Read Full License 


\section{Abstract}

Background: The yak (Bos grunniens) is a crucial resource to supply meat and milk to the people localized in Qinghai-Tibetan plateau area. To identify IncRNAs regulating metabolism in yak, this work adopted transcriptome method to simultaneously profile mRNAs and IncRNAs of liver in yak under three representative age (LD: Liver 1 Day, LM: Liver 15 Months, LY: Liver 5 Years) conditions.

Result: Of 288 differentially expressed IncRNAs, function-oriented selection yield 88 regulated metabolically related IncRNAs that were differentially expressed at least two age conditions. These IncRNAs predicted by IncRNA-mRNA correlation analysis to function in various aspects of metabolism. Selected regulations of liver metabolically related IncRNAs were further verified by qRT-PCR.

Conclusion: Combining high throughput RNA-seq screening screens, bioinformatics predictions, IncRNAmRNA correlation analysis and qRT-PCR analysis, this study supports that a class of IncRNAs function as important metabolic regulators and establishes a foundation for further investigating the role of IncRNAs in yak.

\section{Background}

The yak (Bos grunniens) lives throughout the Qinghai-Tibetan plateau in western China at high altitudes where harsh and variable plateau climate exist including low humidity, temperature, and oxygen levels; strong winds and ultraviolet radiation. The yak has adapted to thrive under these harsh conditions, where few other livestock could survive. The yak is a crucial resource to supply meat and milk to the people localized in Qinghai-Tibetan plateau area. For these reasons, the yak is one of the most critical domestic animals for the 6.5 million Tibetans [1]. Based on the fact that the yak represents the primary source of meat and milk for the people of this region, it is imperative to understand the genetic makeup of the yak and how this can influence its development.

While, metabolic process is one of the most basic activity which is related to all fundamental biological and physiological activities in all animals [2]. Liver, the most important metabolic organs, engage in continuous dialogs to regulate overall metabolic balance by endocrine factors. And it is also a complex digestive gland in ruminants including yak and plays a critical role in the metabolism of substances. More importantly, during the developmental period, liver plays a critical role [3-5]. Dorland et al. previously reported the period of transition from new born to young cattle involved considerable metabolic adaptation in dairy cows and the liver [3]. Therefore, in the present study, the liver of new born (1 day), young yak (15 months) and mature yak (5 years) were chosen to be sampled.

Although a lot of work has been done in studying the regulation of individual metabolic pathways in recent years, there are still quite a few unknow fields in the complicated regulatory networks that control metabolic physiology. It remains difficult to develop useful treatment strategy against metabolic disorders such as diabetes and obesity [6-8]. Nearly two-thirds of transcripts are noncoding RNAs, mainly from regions previously considered as junk gene [9]. Among the noncoding RNAs identified so far, 
IncRNA is the longest and most difficult to understand, which are transcripts of $200 \mathrm{nt}$ or longer that lack protein coding potential. LncRNAs have been recognized in all model organisms [10], and their number has been increasing continuously [11-13]. It has been shown that IncRNAs have an influence on several aspects of cellular function, including chromatin modification and transcription regulation, RNA stability, and translational control [14, 15]. Evolution studies have shown that more than 1,000 IncRNAs may have conservative functions in mammals [16]. There were also many reports in bovine indicates IncRNAs function in embryo development [17], skeletal muscle [18] and mammary glands [19]. In mouse, in silico method had been used to identify functional IncRNAs in metabolic process [20]. However, it is still unknown fields in ruminant animals like bovine or yak.

Therefore, in order to systemically illustrate the importance of IncRNAs in the homeostasis of metabolism of yak, this work combined a high throughput RNA-seq screening of IncRNAs transcripts from liver of yak in diverse ages to establish a comprehensive workflow to determine functional IncRNAs in metabolic regulation. LncRNA-mRNA network analysis is used to predict IncRNAs function, and qRT-PCR analysis connects IncRNAs with specific metabolic pathways. In conclusion, our research supports IncRNAs as a critical composition of metabolism and provides a fundation for systemically identifying and predicting functional IncRNAs that regulate the homeostasis of metabolism.

\section{Result}

\section{Dynamic regulation of IncRNAs and mRNAs in liver under different age conditions}

Both mRNA and IncRNA transcripts were detected in our study, current expression profile could compare the transcripts of IncRNAs and mRNAs at different developmental age stages in the yak liver. Hierarchical cluster analysis was performed on all expressed transcripts, which showed the mRNAs expression profiles clearly divide all samples into three different groups according to age stages ( 1 day, 15 months, 5 years) and these samples were closely clustered together in each group of different developmental age stages (Fig. 1A, top left). But what is interesting to us is that an almost identical sample aggregation pattern showed in the result of IncRNAs (Fig. 1A, top right), which indicates that expression profiles of IncRNAs could be used as a signal indicating similar to those of protein-encoded mRNAs. Using the RNAseq method, 35216 mRNAs and 10073 IncRNAs transcripts were detected from transcripts in the yak livers at three different developmental age stages (LD: Liver 1 Day, LM: Liver 15 Months, LY: Liver 5 Years) (Fig. 2B).

In addition, all samples were divided into diverse groups for both mRNAs and IncRNAs through PCAs analysis on all regulated transcripts (Fig. 1C), which indicate that regulated IncRNAs and mRNAs transcripts might coordinate related biological processes. Furthermore, to determine their functional connections, analysis of the network with IncRNA-mRNA through related analysis of samples were conducted. 433 mRNAs and 152 IncRNAs were identified to be regulated by LD versus LM, 412 mRNAs 


\section{Functional analysis of differentially expressed genes (DEGs) in each age condition of yak liver}

At $L D$ versus $L M$, the DEGs of many functional Gene ontology (GO) categories enriched for metabolism, ion binding, developmental process and so on. While in KEGG pathway analysis, DEGs were also enriched for metabolism, biosynthesis, ECM-receptor interaction and so on (Fig. 2A). At LD versus LY, there were functional GO categories enriched for metabolism, tissue remodeling and developmental process and so on. While in the KEGG pathway analysis, DEGs were also enriched for terms like metabolism, biosynthesis, PI3K-Akt signaling pathway and so on (Fig. 2B). Similar functional GO categories of metabolism, biosynthetic process and oxidation reduction process and so on were enriched at $L M$ versus LY. While in the KEGG pathway analysis, DEGs were also enriched for terms like metabolism, biosynthesis, focal adhesion and so on (Fig. 2C). All these results suggest metabolism related function were developed during the aged process of yak (Fig. 2).

\section{Functional prediction of metabolically related differentially expressed IncRNAs by IncRNA-mRNA co-expression correlation under at least two age conditions}

By correlation of IncRNA-mRNA expression interaction network, dynamically regulated IncRNAs were predicted their function at different developmental ages. 88 non-redundant IncRNAs regulated at least two of the three age conditions were selected from the totally 288 regulated differentially expressed IncRNAs (Fig. 1D). Furthermore, to detect the specific function of these metabolically associated IncRNAs as example, six metabolically related IncRNAs were selected for further analysis (Fig. 3).

Taking six IncRNAs as examples to make the functional analysis, correlation analysis predicts their role in metabolism or cell differentiation. ENSBMUG00000000490 and XLOC_045379 were predicted to be related with fat single organism metabolic process (Fig. 3A and 3B), and XLOC_021536 and XLOC_041441 associated with collagen metabolic processes and protein metabolic processes in liver, respectively (Fig. 3C and 3D). Moreover, ENSBMUG00000026019 and XLOC_183608 have been shown to be enriched in cell proliferation and transport (Fig. 3E and 3F). These results indicate that the established co-expression network can effectively predict the potential metabolic functions of age-regulated and metabolically sensitive IncRNAs. In addition, the dynamic regulation of several randomly selected IncRNAs under different age conditions was confirmed by qRT-PCR, proving the feasibility of this method (Fig. 4).

\section{Discussion}


Integrative IncRNAs function related to metabolic were selected using high-throughput RNA-seq screening, and a workflow for the discovery and characterization of functional IncRNAs were established in yak at different ages. The data obtained in this experiment support that IncRNAs was a crucial component of metabolism. Both the expression profile of IncRNAs and RNAs have change coordinately according to different developmental ages including 1 day, 15 months, and 5 years. Moreover, groups of metabolism relative IncRNAs were regulated in liver by ages, and their expression often changes significantly in yaks at diverse ages, supporting their potential biological significance.

Identifying IncRNAs regulating metabolic and finding its functional properties is still a hard task in animals and only a few IncRNAs could regulate metabolism were reported for now [21-24]. Moreover, it is impossible to directly determine the role of IncRNAs in metabolic process, for the reason of its non-coding feature. In order to get over this difficulty, this article build a functional IncRNAs detection method by interactive analysis of IncRNAs in liver of yaks at different ages. This integrated approach efficiently reduce 288 age-adjusted IncRNAs to 88 putative metabolic IncRNAs which is differentially expressed at least in two age stages.

Only a few previous published papers showed that IncRNAs play a critical role in regulating metabolic pathway in mice [21-24], and certainly there is no report on the function of IncRNAs in yak. In order to understand the processes of yak liver metabolism in depth, more IncRNAs need to be discovered and characterized, and confirming the workflow of IncRNAs could speed up the selection and characterization of metabolic IncRNAs, which offered more perspective for the complex metabolism networks.

Making out the impact of IncRNAs in metabolic pathways at different developmental ages could enhance the unknown fields of complex metabolic physiology. Comparative genomics method had been widely used in revealing potential functions of novel protein-coding genes based on information of homologous genes or protein motifs, but has proved to be noneffective in finding the function of IncRNAs [25]. For most IncRNAs have not been functionally studied, and more importantly, IncRNAs were much more conservative than mRNAs, even between closely related model organisms [26]. Since most IncRNAs are unique to different animals, it is difficult to infer their role according to the sequence matching or evolutionary records [27].

For it is hard to predict functions for IncRNAs, it becomes very difficult to discover and characterize IncRNAs that regulate metabolism. Since metabolism is essential for almost all the biological processes, all organisms, any controlling with vital metabolic pathways is achieved usually through reconnecting metabolic fluxes. Therefore, a strict functional measurement is needed to determine critical points in metabolic regulation. To check the function of IncRNA further in animal models, it is firstly necessary to correctly infer the functional information of IncRNAs. In addition, the results of this experiment can also be beneficial to design targeted and detection methods to determine the unique metabolism in specific IncRNA, which is usually suppressed in complex interaction or compensation situation. Therefore, an efficient method to predict metabolic IncRNAs or connecting IncRNAs to metabolic pathways could significantly accelerate the identification of important IncRNAs metabolic regulators. 


\section{Conclusions}

The present study has illustrated the expression of metabolism-related IncRNAs in different ages of yak and lay a foundation for future finding and characterizing metabolism relative IncRNAs. Potential specific IncRNAs metabolic regulators with possible functions has also been predicted. This study could provide useful information for revealing the metabolic functions of IncRNAs in yak liver.

\section{Methods}

\section{Animal}

Totally nine Maiwa yaks from Yak Technology Park (Hongyuan County of Sichuan Province in China) at diverse age conditions ( 1 day, 15 months and 5 years old) were used in this study and each developmental age stage had three yaks. The experimental animals were healthy and under the same management. Yaks were weighing $10.55 \sim 14.24 \mathrm{~kg}, 96.38 \sim 101.37 \mathrm{~kg}$ and $240.73 \sim 296.36 \mathrm{~kg}$. All yaks were stunned with a captive bolt pistol (Cash 8000 Model Stunner, 0.22 calibre, 4.5 grain cartridge) to ameliorate the suffering of the animals prior to their humane killing, following which exsanguination via a transverse incision of the neck was carried out in the slaughterhouse. Then, the liver tissues were excised immediately and rapidly stored in liquid nitrogen until RNA isolation. Yaks with different age were established for profiling the transcriptomes in liver tissue.

\section{RNA quantification and qualification}

RNAiso Plus (TaKaRa, Japan) was used to extract total liver tissue RNA. The extent of RNA degradation and contamination was monitored on $1 \%$ agarose gels. RNA purity was checked using the NanoPhotometer ${ }^{\circledR}$ spectrophotometer (IMPLEN, CA, USA). RNA concentration was measured using Qubit ${ }^{\circledR}$ RNA Assay Kit in Qubit ${ }^{\circledR}$ 2.0 Flurometer (Life Technologies, CA, USA). RNA integrity was assessed using the RNA Nano 6000 Assay Kit of the Bioanalyzer 2100 system (Agilent Technologies, CA, USA). If the $\mathrm{OD}_{260} / 280$ value of total liver tissue RNA is between $1.8 \sim 2.2$, the quality inspection is qualified (Supplementary Table 1) and the next analysis can be carried out.

\section{Library preparation for high-throughput sequencing}

A total amount of $3 \mu \mathrm{g}$ RNA per sample was used as input material for the RNA sample preparations. A chain-specific library was constructed by removing rRNA, that was, sequencing libraries were generated using NEBNext® UltraTM RNA Library Prep Kit for Illumina ${ }^{\circledR}$ (NEB, USA) [28]. Briefly, mRNAs were purified from total RNA using poly-T oligo-attached magnetic beads. First strand cDNA was synthesized using random hexamer primer and RNase $\mathrm{H}$. Second strand $\mathrm{CDNA}$ synthesis was subsequently performed using DNA Polymerase I and RNase $\mathrm{H}$. The purified double-stranded cDNA was subjected to end repair, was added poly-A tail, and was connected to a sequencing adapter. In order to select cDNA fragments of 
preferentially 150 200 bp in length, the library fragments were purified with AMPure XP system (Beckman Coulter, Beverly, USA). Then $3 \mu \mathrm{L}$ USER enzymes (NEB, USA) were used to degrade the second strand of U-containing CDNA, and perform PCR amplification to obtain the library. After the library was constructed, Qubit2.0 Fluorometer (Invitrogen, USA) was used for preliminary quantification. PCR products were purified (AMPure XP system) and library quality was assessed on the Agilent Bioanalyzer 2100 system. qRT-PCR (Bio-Rad, USA) was used to accurately quantify the effective concentration of the library to ensure library quality. The library preparations were sequenced on an Illumina Hiseq platform and $150 \mathrm{bp}$ paired-end reads were generated.

\section{Transcriptome sequencing data analysis}

The quality-controlled Clean Reads was compared with the yak reference genome (BosGru_v2.0: ftp://ftp.ensembl.org/pub/release-97/fasta/bos_mutus/) quickly and accurately by the HISAT2 v2.0.5 software (http://ccb.jhu.edu/software/hisat2) to obtain Mapping Reads for subsequent analysis [29]. At the same time, the comparison results were evaluated for quality. By analyzing the different regions and chromosome distributions of Mapping Reads in the reference genome, to obtain the comparison efficiency and mapping information about Mapping Reads for each sample [30]. The new transcripts for Mapping Reads were assembled and quantified by StringTie (1.3.3b) software [31]. Sequencing depth and gene length were corrected using FPKM [32]. Differential expression analysis was performed using the DESeq2 R package (1.10.1). DESeq2 provide statistical routines for determining differential expression in digital gene expression data using a model based on the negative binomial distribution. Genes with an adjusted padj<0.05 found by DESeq2 were assigned as differentially expressed [33].

\section{Functional enrichment}

ClusterProfiler (3.4.4) software was used to perform GO function enrichment analysis and KEGG pathway enrichment analysis on differential gene sets, to predict the biological processes and functions that they may participate in, and to make corresponding classification and statistics [34]. All IncRNAs were used for target gene prediction, that was, the target genes of IncRNAs were predicted by the positional relationship (co-location) and expression correlation (co-expression) of IncRNAs and protein-coding genes [35]. Then, functional enrichment analysis (GO / KEGG) was performed on the target genes of differential IncRNAs to predict the main function of IncRNAs.

\section{Real-time fluorescence quantitative PCR}

Measurement of gene expression with qRT-PCR has been applied in our studies. Briefly, total RNA was extracted from liver tissue of each group using a RNAiso Plus (TaKaRa, Japan). RNA was complete without degradation, good quality and high purity, which meet the requirements of subsequent experiments. qRT-PCR was performed in a total volume of $10 \mu \mathrm{L}$ containing $5.2 \mu \mathrm{L}$ of TB Green ${ }^{\mathrm{TM}}$ Premix 


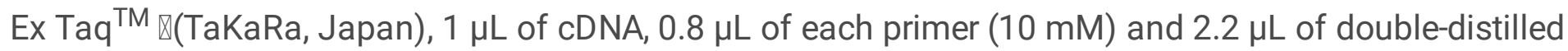
water. The reaction condition used were as follows: $95^{\circ} \mathrm{C}$ for $3 \mathrm{~min}$, followed by 39 cycles of $95^{\circ} \mathrm{C}$ for 10 $\mathrm{s}, 53.4 / 52{ }^{\circ} \mathrm{C}$ ( $\beta$-actin/verification genes) for $20 \mathrm{~s}$ and $72{ }^{\circ} \mathrm{C}$ for $20 \mathrm{~s}$, with the dissolution curve increasing from $0.5^{\circ} \mathrm{C}$ to $95^{\circ} \mathrm{C}$ every $5 \mathrm{~s}$. The assays were performed on a real-time fluorescence quantitative PCR System (Bio-Rad, USA). For each sample, the cycle threshold (CT) values were obtained from three replicates. $\beta$-actin mRNA was employed as an internal reference. The primers used for amplification of target and internal reference genes were presented in Supplementary Table 2. The relative expression levels of target genes were analyzed using the $2^{-\Delta \Delta C T}$ method.

\section{Abbreviations}

RNA: Ribonucleic acid; IncRNAs: Long noncoding RNAs; mRNAs: Messenger RNAs; LD: Liver 1 day; LM: Liver 15 months; LY: Liver 5 years; PCR: Polymerase chain reaction; qRT-PCR: Real-time fluorescence quantitative PCR; RNA-seq: RNA sequence; DNA: Deoxyribonucleic acid; cDNA: Complementary DNA; RNase H: M-MuLV reverse transcriptase; FPKM: Fragments per kilobase of transcript sequence per millions base pairs sequenced; padj: Corrected p-value for multiple hypothesis test; GO: Gene ontology; KEGG: Kyoto encyclopedia of genes and genomes; PCAs: Principal-component analysis; ECM: Extracellular matrix; PI3K-Akt: Phosphatidylinositide 3-kinases-serine/threonine kinase

\section{Declarations}

\section{Acknowledgements}

The authors are grateful to the Novogene Co., Ltd. for providing Illumina Hiseq platform.

\section{Authors' contributions}

LW and XL conceived and designed this study. WX and FF performed experiments, analyzed data and wrote the manuscript. FF and JG collected sample collection. LW and XL contributed to the revisions. All authors reviewed and approved the final manuscript.

\section{Funding}

This work was supported by the National 13th Five-Year Plan Key R \& D Initiative Project (2018YFD0502304) and the Second Comprehensive Scientific Expedition Research Project on the Qinghai-Tibet Plateau (2019QZKK0302) in purchasing yaks, the Sichuan Province studying abroad Scholar Science and Technology Activities Merits Funding Project (2019) and the National Beef Yak Industry Technology System (CARS-37) in sample collection, the Sichuan Science and Technology Support Project (2OYSZH0018 and 16ZC2530) in high throughput RNA-seq, the Young Scientists Fund of the National Natural Science Foundation of China (31900586) and the Central College Youth Funds of 
Southwest Minzu University (2019NQN42) in analysis and interpretation of the data, and the grant from China Scholarship Council (201708510065) played no role in purchasing yaks, sample collection, high throughput RNA-seq, analysis and interpretation of the data.

\section{Availability of data and materials}

All data generated or analysed during this study are included in this published article and its supplementary information files.

\section{Ethics approval and consent to participate}

All animal experiments were conducted according to the regulations for the Administration of Affairs Concerning Experimental Animals (Ministry of Science and Technology, China, revised in June 2004) and approved by the Institution Animal Care and Use Committee in the Southwest Minzu University, Chengdu, China.

\section{Consent for publication}

All of the authors have approved the final version of the manuscript, agree with this submission to BMC Genomics.

\section{Competing interests}

The authors declare that they have no competing interests.

\section{Author details}

${ }^{1}$ Key Laboratory of Qinghai-Tibetan Plateau Animal Genetic Resource Reservation and Utilization, Ministry of Education and Sichuan province, Southwest Minzu University, Chengdu 610041, China;

${ }^{2}$ Sichuan Academy of Grassland Sciences, Chengdu 611731, China

\section{References}

1. Harris RB, Pletscher DH, Loggers $\mathrm{CO}$, et al. Status and trends of Tibetan plateau mammalian fauna, Yeniugou. China Biol Conservation. 1999;87:13-9. https://doi.org/10.1016/s0006-3207(98)00046-9.

2. Lempradl A, Pospisilik JA, Penninger JM. Exploring the emerging complexity in transcriptional regulation of energy homeostasis. Nat Rev Genet. 2015;16(11):665-81.

https://doi.org/10.1038/nrg3941. 
3. Dorland HA van, Richter S, Morel I, et al. Variation in hepatic regulation of metabolism during the dry period and in early lactation in dairy cows. J Dairy Sci. 2009;92(5):1924-40. https://doi.org/10.3168/jds.2008-1454.

4. Graber M, Kohler S, Kaufmann T, et al. A field study on characteristics and diversity of gene expression in the liver of dairy cows during the transition period. J Dairy Sci. 2010;93(11):5200-15. https://doi.org/10.3168/jds.2010-3265.

5. Schlegel G, Ringseis R, Keller J, et al. Changes in the expression of hepatic genes involved in cholesterol homeostasis in dairy cows in the transition period and at different stages of lactation. $J$ Dairy Sci. 2012;95(7):3826-36. https://doi.org/10.3168/jds.2011-5221.

6. Eckel RH, Alberti KG, Grundy SM, et al. The metabolic syndrome. Lancet. 2010;375(9710):181-3. https://doi.org/10.1016/S0140-6736(09)61794-3.

7. Popkin BM, Adair LS, Ng SW. Global nutrition transition and the pandemic of obesity in developing countries. Nutr Rev. 2012;70(1):3-21. https://doi.org/10.1111/j.1753-4887.2011.00456.x.

8. Zimmet PZ, Magliano DJ, Herman WH, et al. Diabetes: a 21st century challenge. Lancet Diabetes Endocrinol. 2014;2(1):56-64. https://doi.org/10.1016/S2213-8587(13)70112-8.

9. 10.1186/gb-2006-7-s1-s4

Harrow J, Denoeud F, Frankish A, et al. GENCODE: producing a reference annotation for ENCODE. Genome Biol. 2006; 7(Suppl 1): S4.1-S4.9. https://doi.org/10.1186/gb-2006-7-s1-s4.

10. Marques AC, Ponting CP. Intergenic IncRNAs and the evolution of gene expression. Curr Opin Genet Dev. 2014;27:48-53. https://doi.org/10.1016/j.gde.2014.03.009.

11. Cabili MN, Trapnell C, Goff L, et al. Integrative annotation of human large intergenic noncoding RNAs reveals global properties and specific subclasses. Genes Dev. 2011;25(18):1915-27. https://doi.org/10.1101/gad.17446611.

12. Guttman M, Amit I, Garber M, et al. Chromatin signature reveals over a thousand highly conserved large non-coding RNAs in mammals. Nature. 2010;458(7235):223-7. https://doi.org/10.1038/nature07672.

13. Luo HT, Sun SL, Li P, et al. Comprehensive characterization of 10,571 mouse large intergenic noncoding RNAs from whole transcriptome sequencing. PLoS One. 2013;8(8):e70835. https://doi.org/10.1371/journal.pone.0070835.

14. Geisler S, Coller J. RNA in unexpected places: long non-coding RNA functions in diverse cellular contexts. Nat Rev Mol Cell Biol. 2013;14(11):699-712. https://doi.org/10.1038/nrm3679.

15. Batista PJ, Chang HY. Long noncoding RNAs: cellular address codes in development and disease. Cell. 2013;152(6):1298-307. https://doi.org/10.1016/j.cell.2013.02.012.

16. Hezroni H, Koppstein D, Schwartz MG, et al. Principles of long noncoding RNA evolution derived from direct comparison of transcriptomes in 17 species. Cell Rep. 2015;11(7):1110-22. https://doi.org/10.1016/j.celrep.2015.04.023.

17. Caballero J, Gilbert I, Fournier E. et.al. Exploring the function of long non-coding RNA in the development of bovine early embryos. Reprod Fertil Dev. 2014;27(1):40-52. 
https://doi.org/10.1071/RD14338.

18. Jin CF, Li Y, Ding XB, et al. Inc133b, a novel, long non-coding RNA, regulates bovine skeletal muscle satellite cell proliferation and differentiation by mediating miR-133b. Gene. 2017;630:35-43. https://doi.org/10.1016/j.gene.2017.07.066.

19. Tong C, Chen QL, Zhao LL, et al. Identification and characterization of long intergenic noncoding RNAs in bovine mammary glands. BMC Genom. 2017;18(1):468. https://doi.org/10.1186/s12864017-3858-4.

20. Yang L, Li P, Yang WJ, et al. Integrative transcriptome analyses of metabolic responses in mice define pivotal IncRNA metabolic regulators. Cell Metab. 2016;24(4):627-39. https://doi.org/10.1016/j.cmet.2016.08.019.

21. Li P, Ruan XB, Yang L, et al. A liver-enriched long non-coding RNA, IncLSTR, regulates systemic lipid metabolism in mice. Cell Metab. 2015;21(3):455-67. https://doi.org/10.1016/j.cmet.2015.02.004.

22. Zhao XY, Lin JD. Long noncoding RNAs: A new regulatory code in metabolic control. Trends Biochem Sci. 2015;40(10):586-96. https://doi.org/10.1016/j.tibs.2015.08.002.

23. Lan X, Yan JD, Ren J, et al. A novel long noncoding RNA Lnc-HC binds hnRNPA2B1 to regulate expressions of Cyp7a1 and Abca1 in hepatocytic cholesterol metabolism. Hepatology. 2016;64(1):58-72. https://doi.org/10.1002/hep.28391.

24. Sallam T, Jones MC, Gilliland T, et al. Feedback modulation of cholesterol metabolism by the lipidresponsive non-coding RNA LeXis. Nature. 2016;534(7605):124-8. https://doi.org/10.1038/nature17674.

25. Ulitsky I, Bartel DP. lincRNAs: genomics, evolution, and mechanisms. Cell. 2013;154(1):26-46. https://doi.org/10.1016/j.cell.2013.06.020.

26. Kutter C, Watt S, Stefflova K, et al. Rapid turnover of long noncoding RNAs and the evolution of gene expression. PLoS Genet. 2012;8(7):e1002841. https://doi.org/10.1371/journal.pgen.1002841.

27. Necsulea $A$, Soumillon $M$, Warnefors $M$, et al. The evolution of IncRNA repertoires and expression patterns in tetrapods. Nature. 2014;505(7485):635-40. https://doi.org/10.1038/nature12943.

28. Parkhomchuk D, Borodina T, Amstislavskiy V, et al. Transcriptome analysis by strand-specific sequencing of complementary DNA. Nucleic Acids Res. 2009;37(18):e123. https://doi.org/10.1093/nar/gkp596.

29. Kim D, Langmead B, Salzberg SL. HISAT: a fast spliced aligner with low memory requirements. Nat Methods. 2015;12(4):357-60. https://doi.org/10.1038/nmeth.3317.

30. Mortazavi A, Williams BA, McCue K, et al. Mapping and quantifying mammalian transcriptomes by RNA-SEq. Nat Methods. 2008;5(7):621-8. https://doi.org/10.1038/nmeth.1226.

31. Pertea M, Pertea GM, Antonescu CM, et al. StringTie enables improved reconstruction of a transcriptome from RNA-seq reads. Nat Biotechnol. 2015;33(3):290-5. https://doi.org/10.1038/nbt.3122. 
32. Bray NL, Pimentel H, Melsted P, et al. Near-optimal probabilistic RNA-seq quantification. Nat Biotechnol. 2016;34(5):525-7. https://doi.org/10.1038/nbt.3519.

33. Love Ml, Huber W, Anders S. Moderated estimation of fold change and dispersion for RNA-seq data with DESeq2. Genome Biol. 2014;15(12):550. https://doi.org/10.1186/s13059-014-0550-8.

34. Yu G, Wang LG, Han Y, et al. clusterProfiler: an R package for comparing biological themes among gene clusters. OMICS. 2012;16(5):284-7. https://doi.org/10.1089/omi.2011.0118.

35. Liao Q, Liu C, Yuan X, et al. Large-scale prediction of long non-coding RNA functions in a coding-noncoding gene co-expression network. Nucleic Acids Res. 2011;39(9):3864-78. https://doi.org/10.1093/nar/gkq1348.

\section{Figures}


A
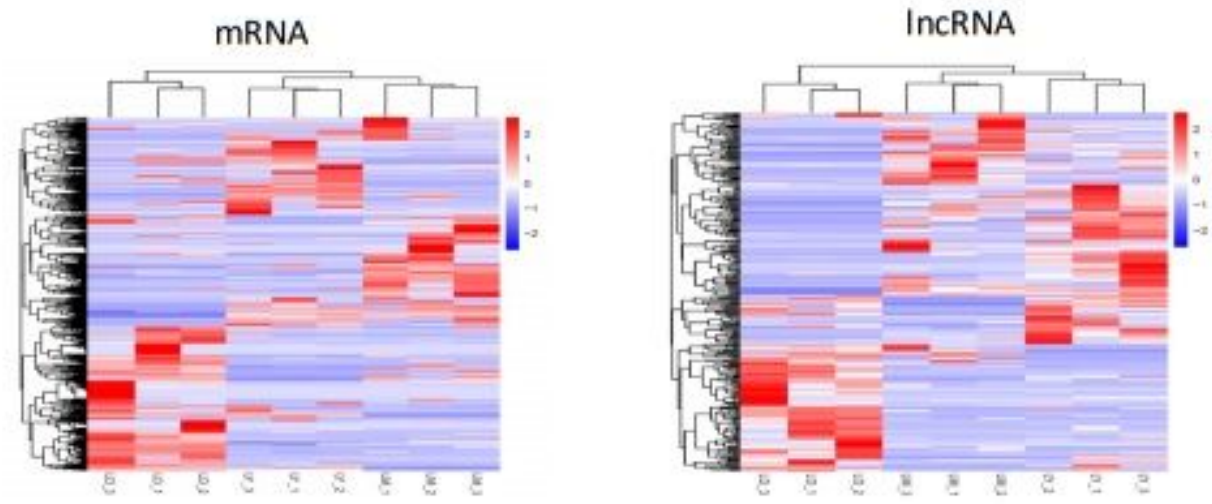

B

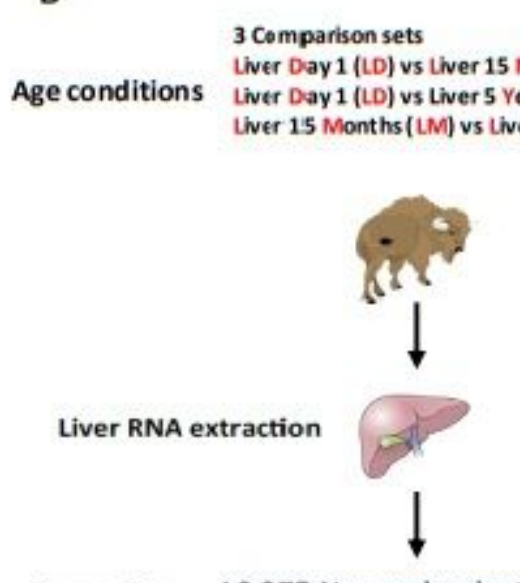

Expression 10,073 Non-redundant IncRNA Profiling 35,216 Coding transcripts
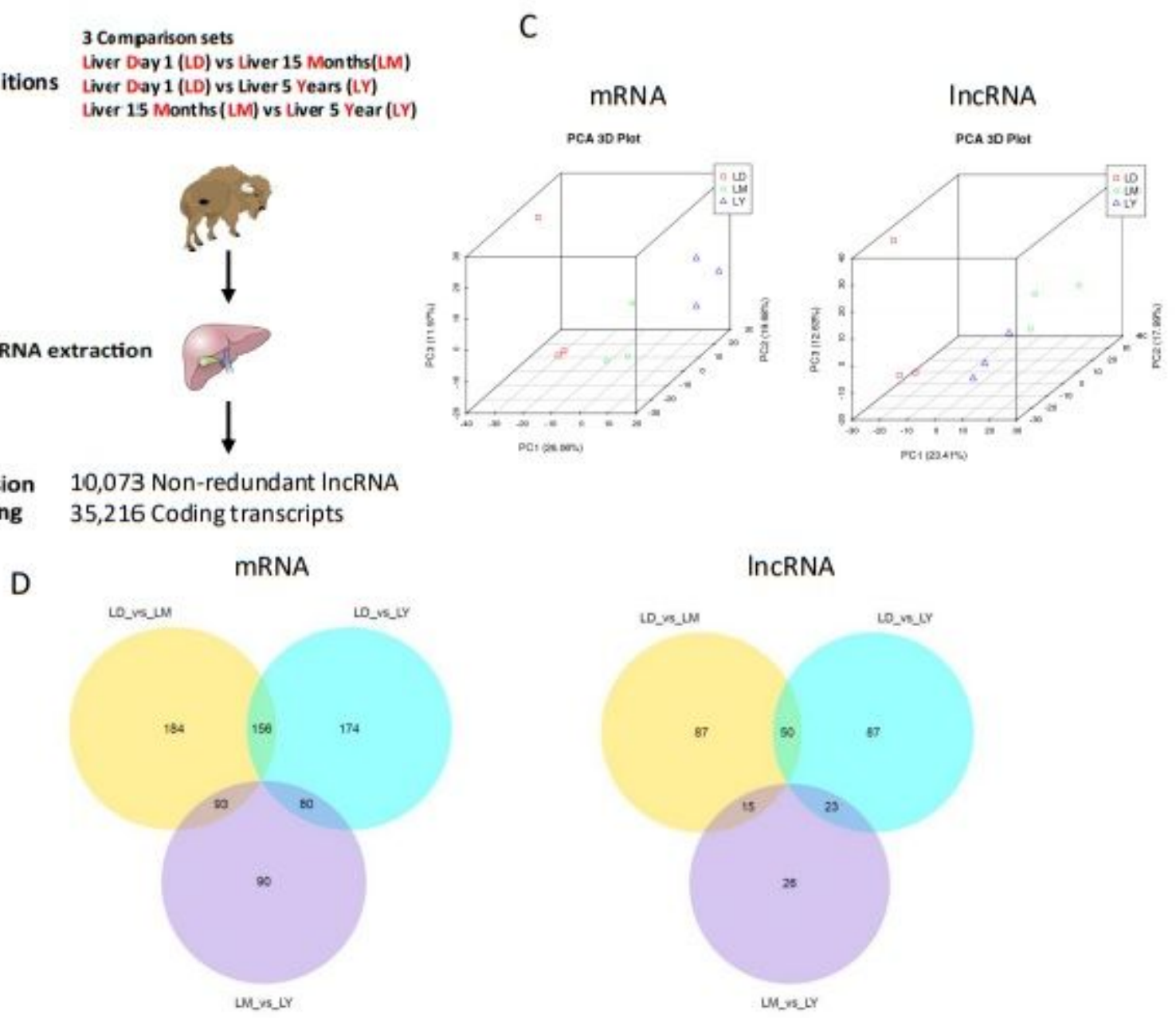

IncRNA

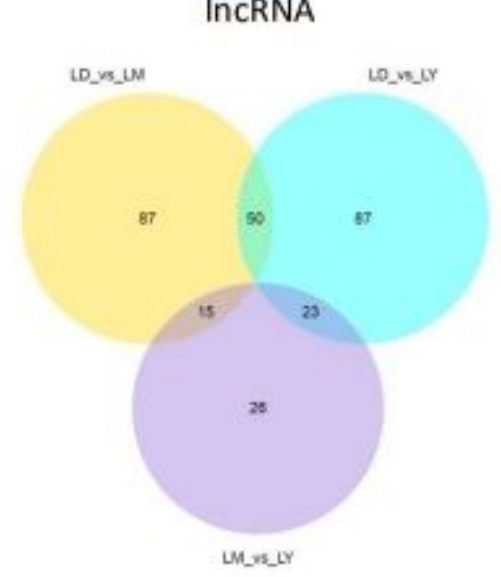

\section{Figure 1}

Dynamic Regulation of LncRNAs and mRNAs in Key Metabolic Organs under Multiple Age Conditions (A) Hierarchical clustering (top) and PCAs (bottom) of differentially expressed mRNAs (left) and IncRNAs (right) in yak livers at different age conditions. The differentially expressed genes were defined by oneway ANOVA to be significantly different. (B) Experimental outline. Each metabolic condition contains three yak, and the expression profiles of mRNAs or IncRNAs from 9 liver samples were analyzed. (C) PCAs (bottom) of differentially expressed mRNAs (left) and IncRNAs (right) in yak livers. (D) Numerical distribution of age-specifically and commonly regulated mRNAs (left) and IncRNAs (right) in liver. 
A
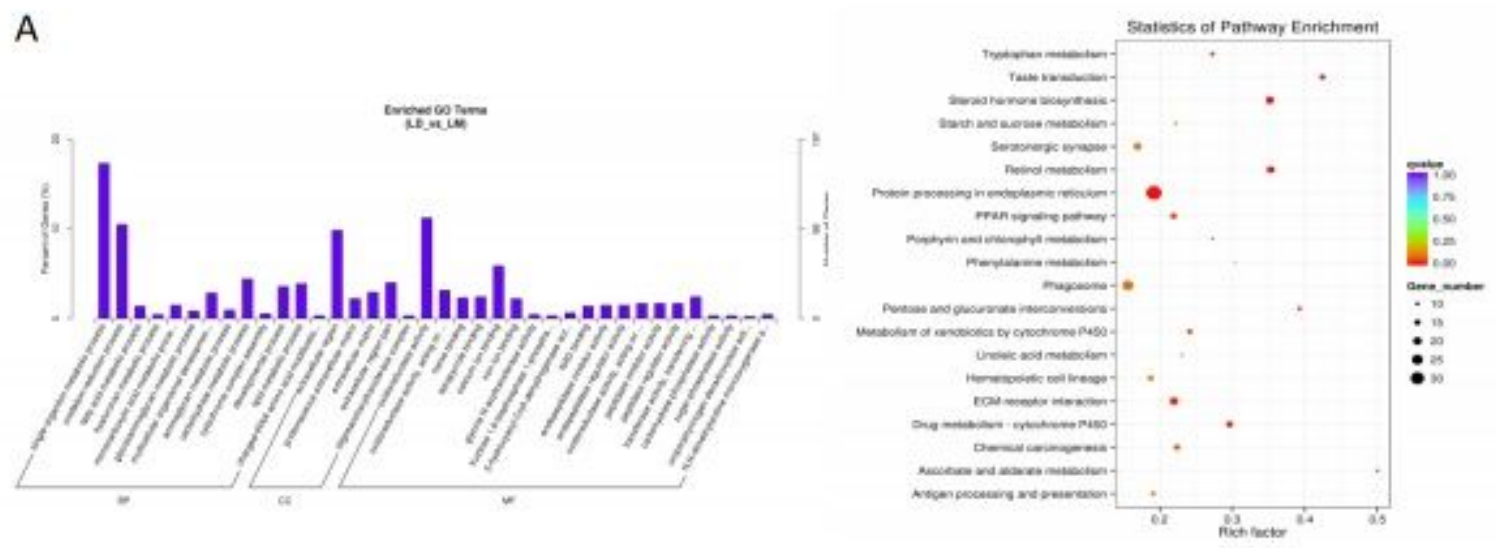

B
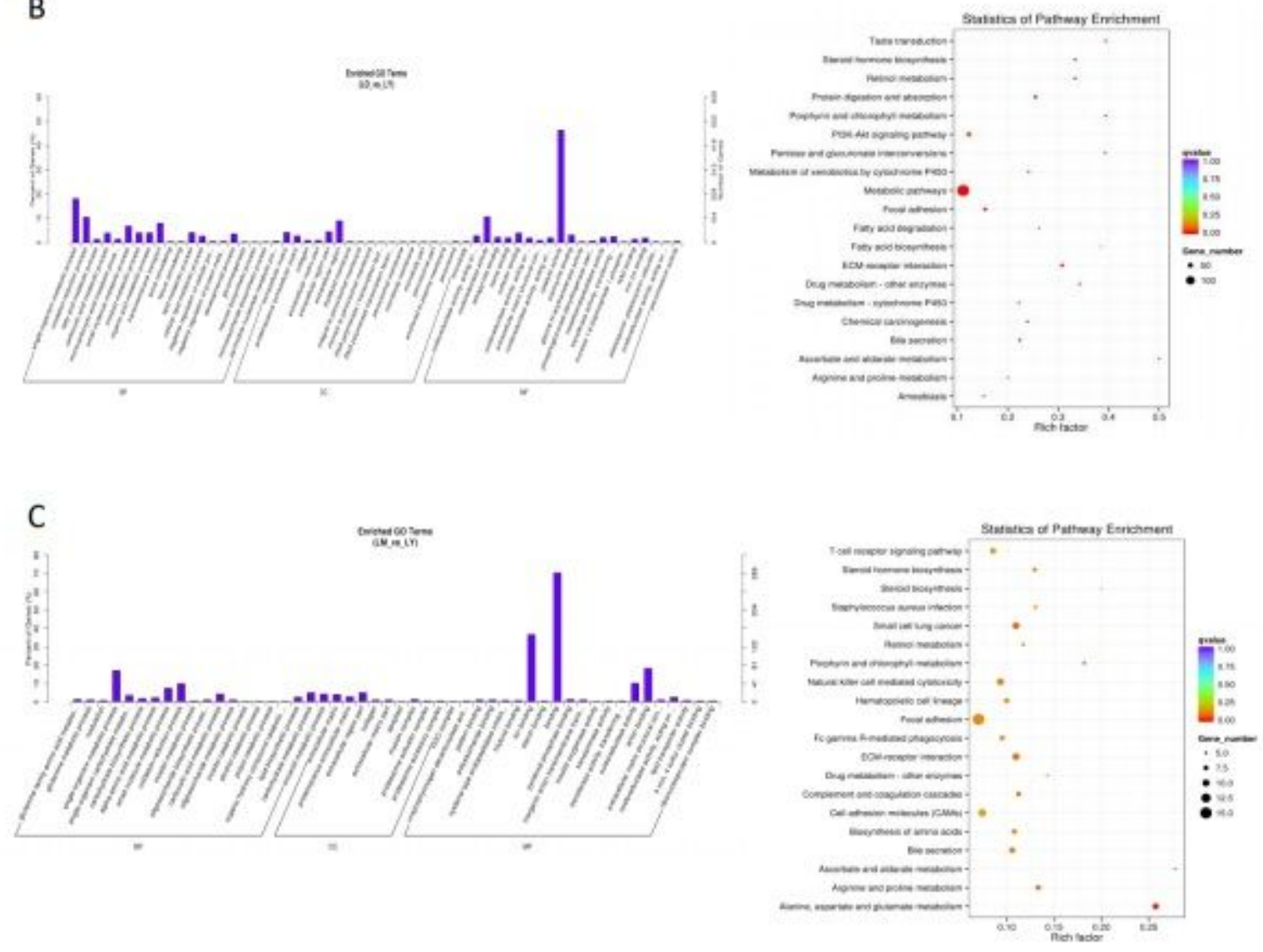

Figure 2

gene ontology (GO) and KEGG pathway terms for differentially expressed mRNAs in each age condition in liver (A) Gene ontology (left) and KEGG pathway(right) terms for differentially expressed mRNAs of LD vs LM in yak liver. (B) Gene ontology (left) and KEGG pathway(right) terms for differentially expressed mRNAs of LD vs LY in yak liver. (C) Gene ontology (left) and KEGG pathway(right) terms for differentially expressed mRNAs of LM vs LY in yak liver 
A

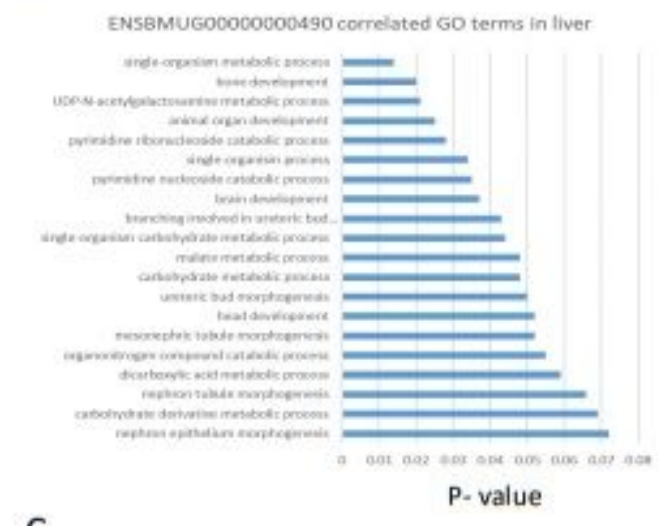

C

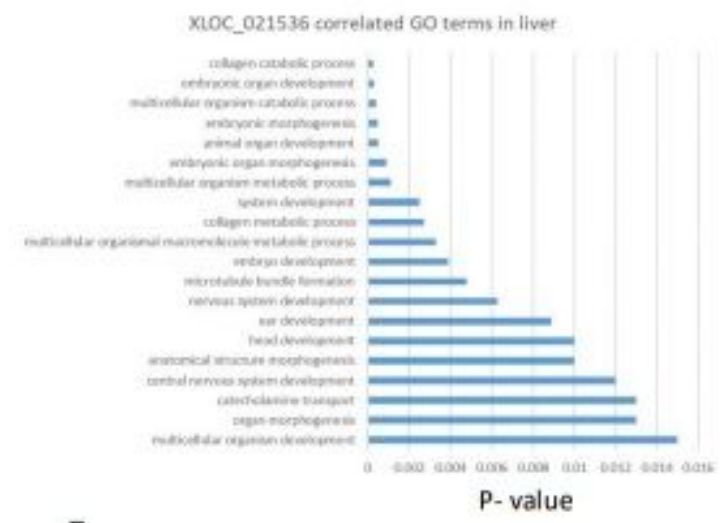

\section{E}

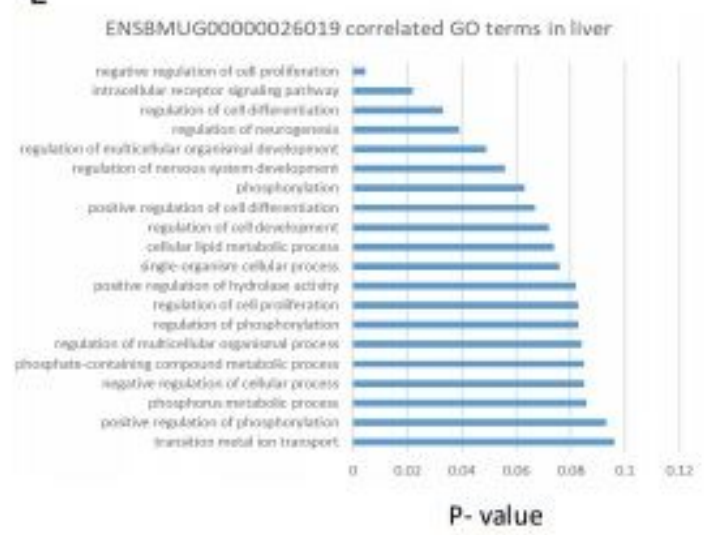

B

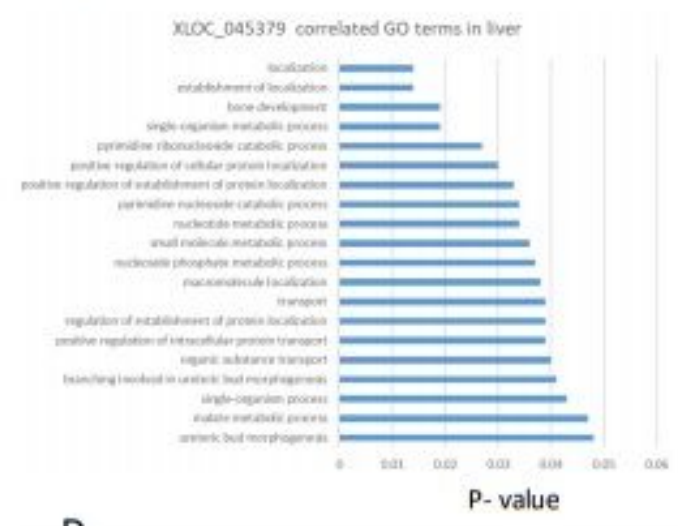

D

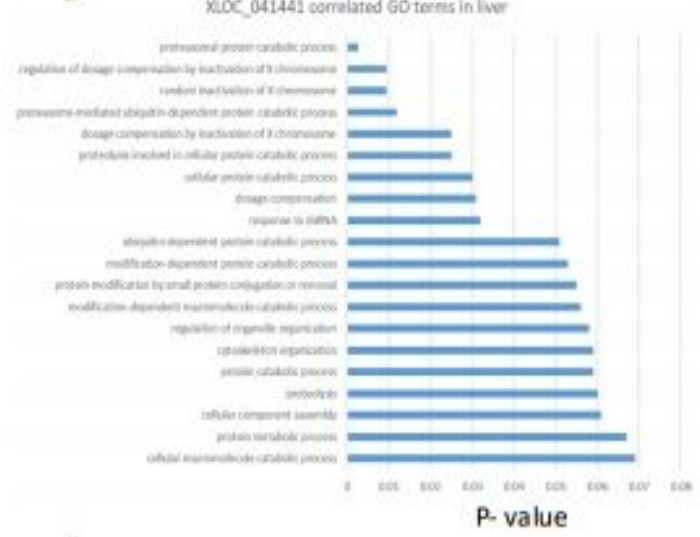

F

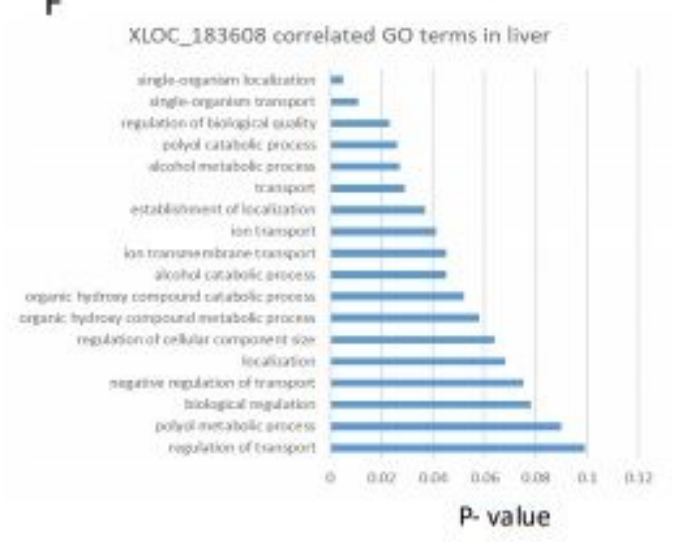

\section{Figure 3}

Functional Prediction of Metabolically related Differentially expressed LncRNAs by LncRNA-mRNA Coexpression Correlation under at least two Age Conditions (A) Gene ontology terms for differentially expressed IncRNA ENSBMUG00000000490 in yak liver. (B) Gene ontology terms for differentially expressed IncRNA XLOC_045379 in yak liver. (C)Gene ontology terms for differentially expressed IncRNA XLOC_021536 in yak liver. (D)Gene ontology terms for differentially expressed IncRNA XLOC_041441 in yak liver. (E) Gene ontology terms for differentially expressed IncRNA ENSBMUG00000026019 in yak liver. (F) Gene ontology terms for differentially expressed IncRNA XLOC_183608 in yak liver. 
A

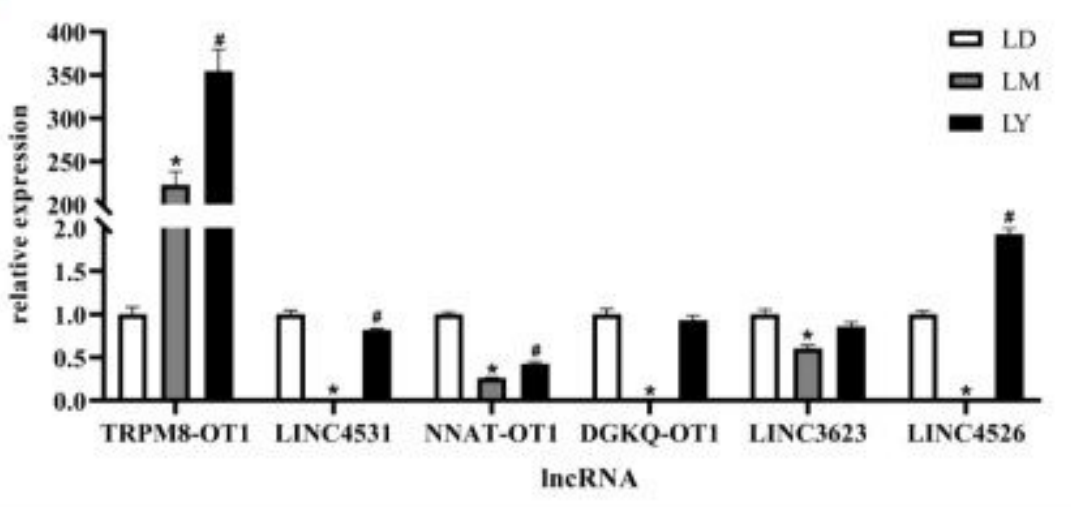

B

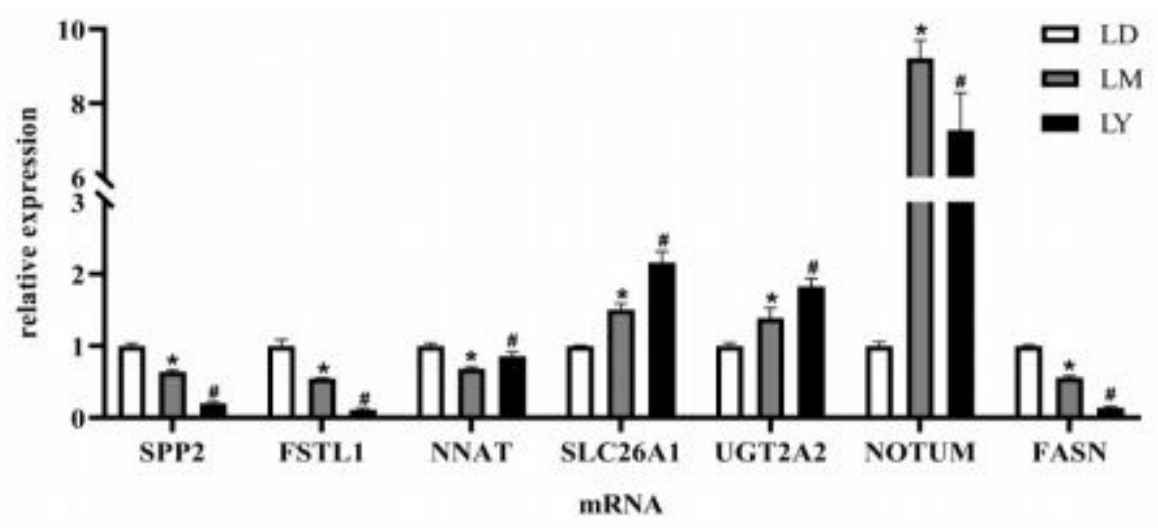

C

\begin{tabular}{cc}
\multicolumn{2}{c}{ mRNA } \\
\hline FPKM value & Correlation between \\
& RNA-seq and FPKM \\
\hline $0.5<$ FPKM $<10$ & $r=0.896$ \\
$10<$ FPKM $<100)$ & $r=0.742$ \\
FPKM $>100$ & $r=0.974$ \\
FPKM $>0.5$ (all) & $r=0.98$ \\
\hline
\end{tabular}

\begin{tabular}{cc}
\multicolumn{2}{c}{ IncRNA } \\
\hline FPKM value & $\begin{array}{c}\text { Correlation between } \\
\text { RNA-seq and FPKM }\end{array}$ \\
\hline $0.001<$ FPKM $<1$ & $\mathrm{r}=0.877$ \\
FPKM $>1$ & $\mathrm{r}=0.982$ \\
FPKM $>0.001$ (all) & $\mathrm{r}=0.983$ \\
\hline
\end{tabular}

\section{Figure 4}

qPCR validation of selected Differentially expressed LncRNAs and mRNAs (A) Validation of IncRNA RNAseq results by quantitative real-time PCR analysis. Expression levels of 6 IncRNAs were quantified. Error bars are SEM, $n=4$. * $P<0.05$ (LD vs LM), \# $p<0.05$ (LD vs $L Y$ ). (B) Validation of mRNA RNA-seq results by quantitative real-time PCR analysis. Expression levels of 7 mRNAs were quantified. Error bars are SEM, $n=4$. * $P<0.05$ (LD vs LM), \# $p<0.05$ (LD vs LY). (C) Pearson correlation of log2-transformd fold changes between FPKM and RNA-seq for differentially expressed mRNA(left) and IncRNAs(right).

\section{Supplementary Files}

This is a list of supplementary files associated with this preprint. Click to download. 
- supplementaryTable.pdf

Page $17 / 17$ 